\title{
水热法制备氧化铬及其常温低浓度一氧化氮催化性能
}

\author{
吴美健 ${ }^{1}$, 高振源 ${ }^{2}$, 袁 静 $^{2}$, 赵昆峰 ${ }^{2}$, 蔡 婷 ${ }^{2}$, 杨 玲 ${ }^{2}$, 张 涛 ${ }^{2}$, 何丹农 ${ }^{1,2}$
}

(1. 上海交通大学 材料科学与工程学院, 上海 200240; 2. 纳米技术及应用国家工程研究中心, 上海 200241)

摘 要: 以十六烷基三甲基溴化铵 (CTAB)为模板剂, 氨水为沉淀剂, 通过水热法并焙烧处理合成氧化铭催化剂。采 用 $X$ 射线衍射分析 $(X R D)$ 、傅里叶变换红外光谱(FT-IR)、X 射线光电子能谱(XPS)和透射电镜(TEM)等技术对催化 剂的物化性质进行了表征, 并考察常温不同 NO 初始浓度下氧化铬催化剂的 NO 氧化性能。结果表明: 在空速为 $60000 \mathrm{~mL} /(\mathrm{g} \cdot \mathrm{h})$ 和 $\mathrm{NO}$ 浓度为 $1 \times 10^{-6}$ 的条件下, $100^{\circ} \mathrm{C}$ 水热温度制备的 $\mathrm{Cr}-100$ 催化剂表现出最优的性能, 常温下 NO 消 除率高达 $90 \%$ 以上并保持 $120 \mathrm{~h}$, 其优异的常温催化性能与催化剂表面较高的 $\mathrm{Cr}^{6+} / \mathrm{Cr}^{3+}$ 摩尔比有关。研究结果表明: 催 化剂的失活主要是由于硝酸根在催化剂表面的累积而导致催化剂活性中心被覆盖, 低浓度下能减缓硝酸根的积累。

关 键 词: 水热法; 常温; 失活; 氧化铬

中图分类号: X511 文献标识码: A

\section{Hydrothermal Fabrication and Catalytic Performance of Chromium Oxide for Low-concentration NO Oxidation at Ambient Temperature}

\author{
WU Mei-Jian ${ }^{1}$, GAO Zhen-Yuan ${ }^{2}$, YUAN Jing ${ }^{2}$, ZHAO Kun-Feng ${ }^{2}$, CAI Ting ${ }^{2}$, YANG Ling ${ }^{2}$, \\ ZHANG Tao ${ }^{2}$, HE Dan-Nong ${ }^{1,2}$
}

(1. School of Materials Science and Engineering, Shanghai Jiao Tong University, Shanghai 200240, China; 2. National Engineering Research Center for Nanotechnology, Shanghai 200241, China)

\begin{abstract}
The chromic oxide catalysts were synthesized by hydrothermal method using CTAB as surfactant and $\mathrm{NH}_{3} \cdot \mathrm{H}_{2} \mathrm{O}$ as precipitant. Physiochemical properties of these catalysts were characterized by X-ray diffraction (XRD), Fourier Transform lufrared specfroscopy (FT-IR), X-ray photoelectron spectroscope (XPS), and transmission electron microscope (TEM). The catalytic NO removal performances for different NO concentrations at room temperature was also investigated. It was shown that under the conditions of space velocity of $60,000 \mathrm{~mL} /(\mathrm{g} \cdot \mathrm{h})$ and $\mathrm{NO}$ concentration of $1 \times 10^{-6}$, sample of Cr-100 exhibited the best catalytic performance, which maintained $120 \mathrm{~h}$ as removal ratio of NO more than $90 \%$. This excellent catalytic performance could be attributed to the surface ions ratio of $\mathrm{Cr}^{6+} / \mathrm{Cr}^{3+}$. The FT-IR analysis indicated that catalyst deactivation was due to the active sites of chromic oxide occupied by nitrates, consistent with the results of XPS. Furthermore, the activity tests in different conditions demonstrated that low NO concentration could slow down the rate of nitrate accumulation and prolong the catalyst lifetime.
\end{abstract}

Key words: hydrothermal; ambient temperature; deactivation; chromic oxide

城市化进程的加快以及机动车保有量的增加使 得汽车尾气排放量急剧增长, 而汽车尾气中含有大量 的氮氧化物 $\left(\mathrm{NO}_{x}\right)^{[1]}$, 对人体健康产生极大危害 ${ }^{[2-4]}$ 。在
隧道和地下停车场等半封闭结构空间，自然通风受 到限制, 加上经三效催化剂处理的汽车尾气中仍残 留部分 $\mathrm{NO}_{x}$, 使隧道和地下停车场成为机动车排放 
污染物最集中的地方之一, 因此急需对半封闭空间 $\mathrm{NO}_{x}$ 实施净化治理。

目前较为广泛的 $\mathrm{NO}_{x}$ 治理技术主要有选择性催

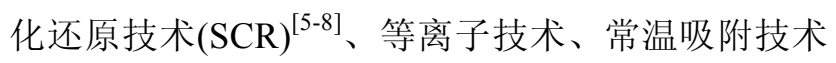
等。SCR 主要消除较高浓度的 $\mathrm{NO}_{x}$, 但需要高温条 件, 难以消除常温低浓度 $\mathrm{NO}_{x}$; 等离子技术所需能 耗较高, 无法全面推广; 常温吸附则受限于饱和吸 附量 ${ }^{[9-10]}$, 且 NO 较难吸附。因此现有技术难以在常 温下消除低浓度 $\mathrm{NO}_{x}$ 。

考虑到半封闭空间中的 $\mathrm{NO}_{x}$ 大部分为 $\mathrm{NO}$, 并 且 $\mathrm{NO}_{2}$ 较 $\mathrm{NO}$ 更容易被碱液吸收, 因此可在常温下 将 $\mathrm{NO}$ 转化为 $\mathrm{NO}_{2}$, 再结合碱液吸收 $\mathrm{NO}_{2}$, 实现 $\mathrm{NO}_{x}$ 的完全消除。其中, 设计在常温下催化 $\mathrm{NO}$ 为 $\mathrm{NO}_{2}$ 的催化剂最为关键。在已报道的 NO 常温氧化催化 剂中, 以锰基催化剂研究为多, 包括单一的氧化锰 和掺杂 $\mathrm{Fe} 、 \mathrm{Ni} 、 \mathrm{Co} 、 \mathrm{Zn}$ 的复合氧化锰基催化剂 ${ }^{[11-13]}$ 。

本课题组 ${ }^{[14]}$ 曾采用沉淀法制备了氧化铬催化 剂, 实现了 NO 常温消除, 当 NO 浓度为 $15 \times 10^{-6}$ 时, 初始效率可达 $100 \%$, 但持续时间过短, 稳定性 不足。在此基础上, 本工作以 CTAB 为模板剂, 硝 酸铬为铬源, 氨水为沉淀剂, 采用水热法制备高效 稳定的常温低浓度 NO 氧化铬催化剂, 采用多种技 术手段对其进行表征。考察了不同水热温度下制备 的氧化铬催化剂在低 NO 浓度下的氧化性能, 并对 NO 常温消除测试前后的催化剂的物化性质进行研 究, 探讨了催化剂失活的原因。

\section{1 实验方法}

\section{1 催化剂的制备}

将 $10 \mathrm{~g}$ 硝酸铬(分析纯, 国药集团)溶解于 $300 \mathrm{~g}$ 去离子水, 待完全溶解后加入 $10 \mathrm{~g} \mathrm{CTAB}$ (分析纯, 国药集团), 用氨水调节 $\mathrm{pH}$ 至 9 , 继续摚拌 $5 \mathrm{~h}$, 转 移至聚四氟乙烯釜中, 置于 $Y^{\circ} \mathrm{C}$ 烘箱中 $(Y=80 、 100$ 、 $120 、 140 、 160)$, 并保持 $20 \mathrm{~h}$ 。冷却至室温后, 抽滤 并分别用热水与乙醇洗涤 3 次, 置于 $50^{\circ} \mathrm{C}$ 烘箱中, 干燥 $12 \mathrm{~h}$ 。所得样品在马弗炉中焙烧, 以 $2^{\circ} \mathrm{C} / \mathrm{min}$ 升至 $300^{\circ} \mathrm{C}$, 并保持 $4 \mathrm{~h}$ 。样品以 $\mathrm{Cr}-Y$ 来表示 4 个不 同水热温度下制备的催化剂, Cr-100U 表示失活后 的 $\mathrm{Cr}-100$ 催化剂。

\section{2 催化剂的表征}

用 Rigaku D/max-2600 PC 型 X 射线衍射分析仪 分析催化剂的结构, 采用 $\mathrm{Cu}$ 靶 $\mathrm{K} \alpha$ 射线 $(\lambda=0.1541 \mathrm{~nm})$, 扫描范围为 $2 \theta=10^{\circ} \sim 80^{\circ}$ 。在 Nicolet 6700 型红外光 谱仪上进行催化剂的 FT-IR 表征, 固体采用 $\mathrm{KBr}$ 压
片法, 液体涂抹在 $\mathrm{KBr}$ 膜上, 扫描波数范围为 400 4000 $\mathrm{cm}^{-1}$ 。在 JEM-2100F 透射电镜上观察催化 剂的微观形貌, 工作电压为 $200 \mathrm{kV}$ 。样品直接分散 在无水乙醇中, 并超声分散 $20 \mathrm{~min}$, 然后滴在镀碳 膜的铜网上并自然干燥。在 Kratos AXIS UltraDLD 上进行催化剂的 XPS 表征, 使用 $\mathrm{AlK} \alpha$ 射线, 工作 电压与工作电流分别为 $15 \mathrm{kV}$ 和 $10 \mathrm{~mA}$ 。

\section{3 催化剂的性能评价}

在常压固定床 $U$ 型反应器( 内径为 $12 \mathrm{~mm}$ )中进行 $\mathrm{NO}$ 常温消除反应性能评价。所用原料气组分: NO 浓 度为 $0 \sim 10 \times 10^{-6}$, 其余为空气, 总流量为 $500 \mathrm{~mL} / \mathrm{min}$, 催化剂质量为 $0.2 \mathrm{~g} \sim 1.0 \mathrm{~g}$ (20 40 目), U 型反应管放 置在 $30^{\circ} \mathrm{C}$ 恒温水浴槽内, 原料气体直接通过催化剂 床层。使用 NO 分析仪(Thermo, Model 42i)进行气体 浓度分析, 仪器的最低检测限为 $0.04 \times 10^{-9}$ 。测试 空速的变化通过改变催化剂质量来实现。 $\mathrm{NO}$ 的消 除率表示为:

$$
\mathrm{NO} \text { 消除率 }=\frac{\mathrm{NO} \text { 进口浓度 }-\mathrm{NO}_{\text {出口浓度 }}}{\mathrm{NO} \text { 进口浓度 }} \times 100 \%
$$

\section{2 结果与讨论}

\section{$2.1 \mathrm{X}$ 射线衍射分析}

图 1 为不同水热温度下制备的催化剂的 XRD 图谱, 从图中可以看到不同催化剂的衍射峰型没有 明显差别, 在 $2 \theta=36^{\circ} 、 55^{\circ}$ 处存在最强峰, 对应均为 氧化铬的六方晶型, 只是衍射峰的强度有所差异。 随着水热温度的上升, 催化剂衍射峰强度在增强, 即 Cr-160 衍射峰最强, Cr- 80 衍射峰较弱。用谢乐公 式计算了不同催化剂的晶粒尺寸, 表 1 显示催化剂 Cr-100 的粒径最小，而催化剂 Cr-160 的晶粒尺寸最 大, 粒径为 $37.6 \mathrm{~nm}$, 晶粒尺寸呈现出随水热温度升 高呈现先减小后增大的变化特点。从 XRD 图谱可 知, Cr-100 的氧化铬晶化程度相对较差。一般来说, 水热法制备催化剂过程中水热温度越高, 催化剂的 晶型相对越完整 ${ }^{[15]}$, 催化剂中晶格缺陷相应越少。 经 NO 常温消除测试后, 失活 Cr-100 催化剂的衍射 峰强度有所增强, 经计算得知 $\mathrm{Cr}-100 \mathrm{U}$ 的晶粒尺寸 由原来 $20.7 \mathrm{~nm}$ 增大到 $22 \mathrm{~nm}$, 这可能是由于在反应过 程中晶粒在失活过程中发生团聚导致晶粒的长大 ${ }^{[16]}$ 。

\section{2 透射电镜分析}

图 2 为两种水热温度下制备的催化剂透射电镜 照片。图 2(b)为 Cr-100 的 HRTEM 照片, 图中存在 $\mathrm{Cr}_{2} \mathrm{O}_{3}$ 的(311)晶面与 $\mathrm{CrO}_{3}$ 的(211)晶面, 表明 $\mathrm{Cr}-100$ 中同时存在 $\mathrm{CrO}_{3}$ 与 $\mathrm{Cr}_{2} \mathrm{O}_{3}$ 。图 2(d)为 $\mathrm{Cr}-160$ 的 


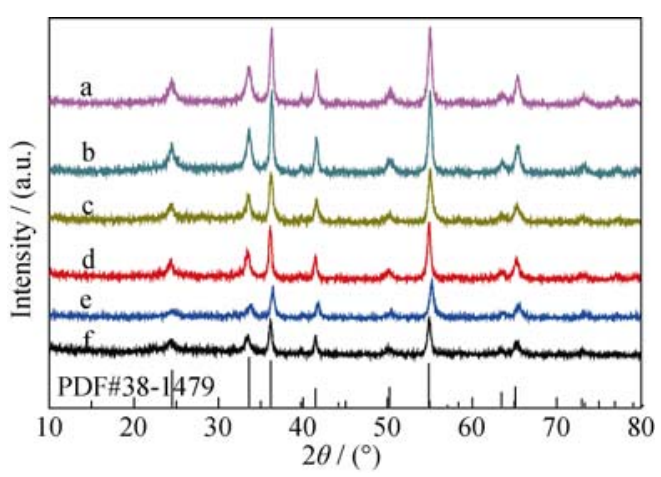

图 1 不同水热温度下制备的催化剂 XRD 图谱

Fig. 1 XRD patterns of catalyst synthesized at various hydrothermal temperatures

(a) $\mathrm{Cr}-160$; (b) $\mathrm{Cr}-140$; (c) $\mathrm{Cr}-120$; (d) $\mathrm{Cr}-100 \mathrm{U}$; (e) $\mathrm{Cr}-100$; (f) $\mathrm{Cr}-80$

HRTEM 照片, 其晶格条纹比较明显, 并主要为 $\mathrm{Cr}_{2} \mathrm{O}_{3}$ 的(311) 晶面, 表明在较高温度下合成的 Cr-160 具有相对完整且比较单一的晶体结构。

\section{$2.3 \mathrm{X}$ 射线光电子能谱分析}

催化剂氧化性能与不同价态 $\mathrm{Cr}$ 离子存在密切 关系, 图 3 为不同水热温度制备催化剂与 NO 消除 测试失活后 Cr-100U 的 XPS 图谱, 在图谱中不同的 结合能峰位置代表 $\mathrm{Cr}$ 不同价态 ${ }^{[17]}$ 。从图 3 中可知 各水热温度下制备的催化剂均含有 $\mathrm{Cr}^{3+}$ 与 $\mathrm{Cr}^{6+}$ 两种 价态, 只是催化剂中的 $\mathrm{Cr}^{6+} / \mathrm{Cr}^{3+}$ 比例存在区别。对 比图 3(a、c、d)和表 1 可知, 随着制备条件水热温 度的升高, $\mathrm{Cr}^{6+}$ 含量不断下降, 其中 $\mathrm{Cr}-100$ 的 $\mathrm{Cr}^{6+} / \mathrm{Cr}^{3+}$ 含量比为 $0.64, \mathrm{Cr}-80$ 与 $\mathrm{Cr}-160$ 的 $\mathrm{Cr}^{6+} / \mathrm{Cr}^{3+}$ 分别为 0.37 与 0.23 。 $\mathrm{Cr}^{6+}$ 的含量与催化剂的性能有
关联, 研究表明催化剂的 NO 常温催化性能与其 $\mathrm{Cr}^{6+}$ 含量呈正相关 ${ }^{[18]}$, XPS 证明 $\mathrm{Cr}^{6+}$ 是影响催化剂 NO 催化活性的重要因素, 还进一步验证透射电镜 的结果, 即高温下趋向形成较单一完整的晶体结 构。对比表 1 中催化剂 Cr-100 常温 NO 消除测试失 活前后的 N/Cr 比, Cr-100 测试前没有 $\mathrm{N}$ 元素的存在, 但 $\mathrm{Cr}-100 \mathrm{U}$ 中的 $\mathrm{N} / \mathrm{Cr}$ 却升至 0.23 , 表明测试过程中 有 $\mathrm{N}$ 元素的产生。由于 $\mathrm{NO}$ 消除测试过程中吸附的 $\mathrm{NO}_{2}$ 在催化剂表面与水生成硝酸根, 在催化剂的表 面沉积, 随着测试进行导致 $\mathrm{N}$ 元素的增加。失活催 化剂中 $\mathrm{N}$ 元素的 XPS 检测结果表明, 硝酸根在催化 剂表面的沉积是催化剂失活的重要影响因素。

XPS 表征结果显示氧化铬并不是单一价态的氧 化物催化剂, 而是两种价态的混合物。图 4 为 Cr-100 试样经 NO 消除测试前后针对 O1s 的 XPS 分析结果, 通过高斯拟合可将 $\mathrm{O} 1 \mathrm{~s}$ 分为三部分 ${ }^{[19]}$ : 一是晶格氧, 存在于晶格表面，其能谱位于 $530.1 \mathrm{eV}$; 二是吸附 氧，结合能为 $531.1 \mathrm{eV}$; 三是所谓的羟基氧，结合能 为 $532.8 \mathrm{eV}$, 一般情况下吸附氧的大量存在对催化 反应起促进作用。经计算, Cr-100 的 NO 消除测试前 吸附氧与晶格氧的比例为 0.76 , 但 Cr-100 失活后其 比例降为 0.57 , 可知吸附氧在测试过程中参与反应, 并被不断消耗。在催化过程中金属原子通过捕捉 $\mathrm{O}_{2}$ 并使 $\mathrm{O}$ 原子活化, 进而增强催化剂的氧化能力。随 着氧化反应的进行, 吸附氧不断被消耗降低。显然 催化反应过程中存在吸附氧的参与, 吸附氧对催化 剂的催化效果有促进作用。
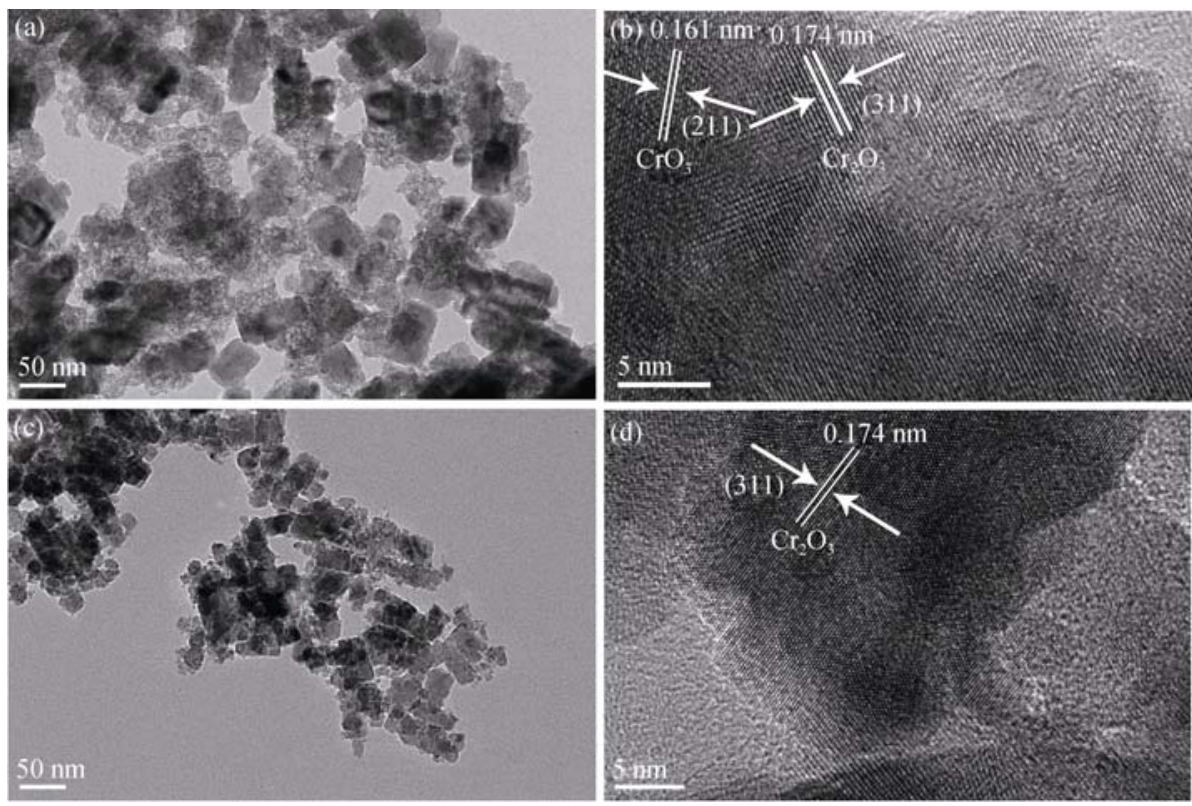

图 2 两种水热温度下制备的催化剂的透射电镜照片

Fig. 2 TEM (a, c) and HETEM (b, d) images of the chromium oxide catalysts synthesized at different hydrothermal temperatures (a) $\mathrm{Cr}-100$; (b) $\mathrm{Cr}-100$; (c) $\mathrm{Cr}-160$; (d) $\mathrm{Cr}-160$ 

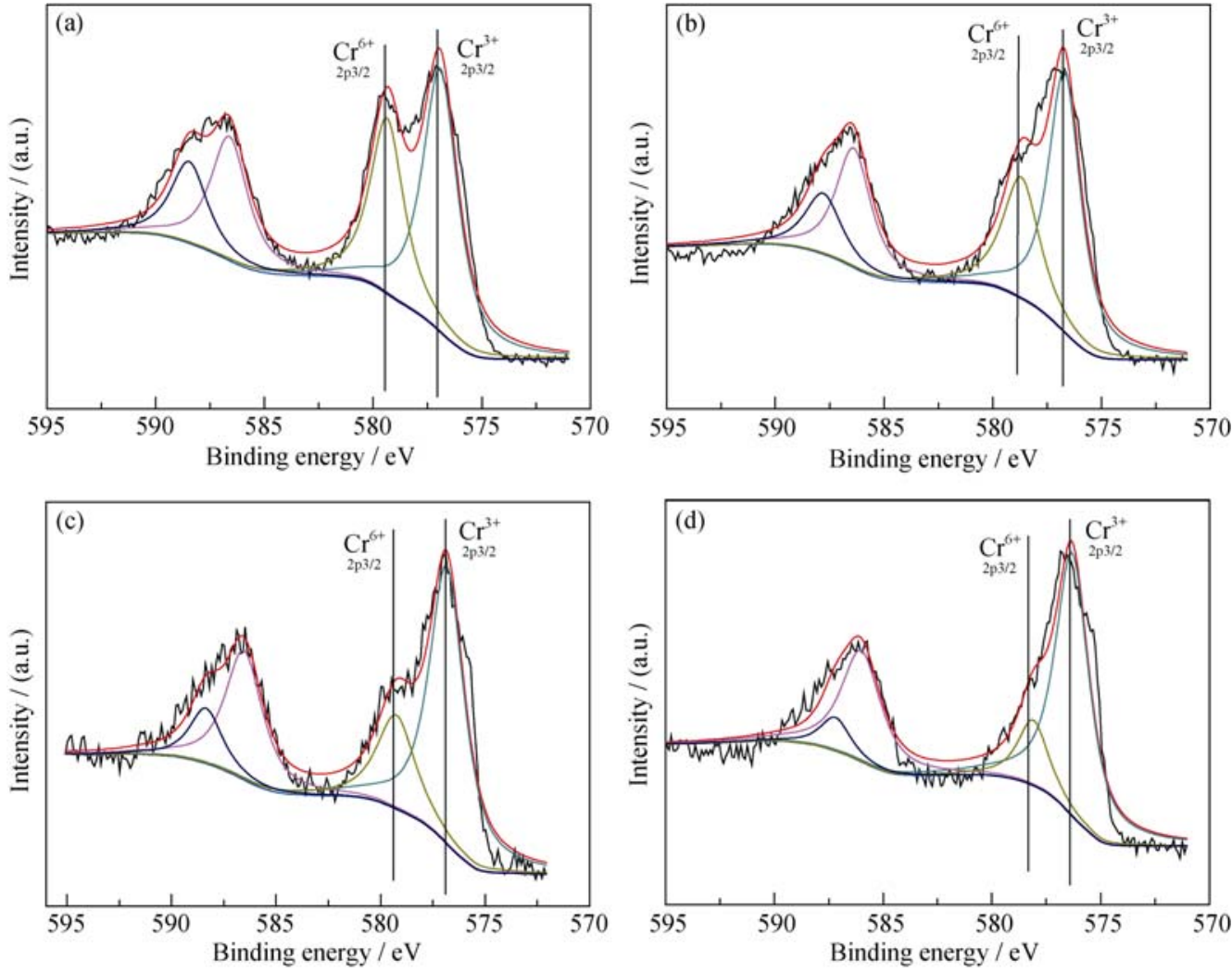

图 3 不同水热温度下制备的催化剂与 NO 消除测试失活后催化剂的 $\mathrm{Cr} 2 \mathrm{p}$ 的 XPS 图谱

Fig. 3 XPS spectra of $\mathrm{Cr} 2 \mathrm{p}$ of fresh samples prepared at different hydrothermal temperatures and catalyst after NO removal test (a) $\mathrm{Cr}-100$; (b) $\mathrm{Cr}-100 \mathrm{U}$; (c) $\mathrm{Cr}-80$; (d) $\mathrm{Cr}-160$
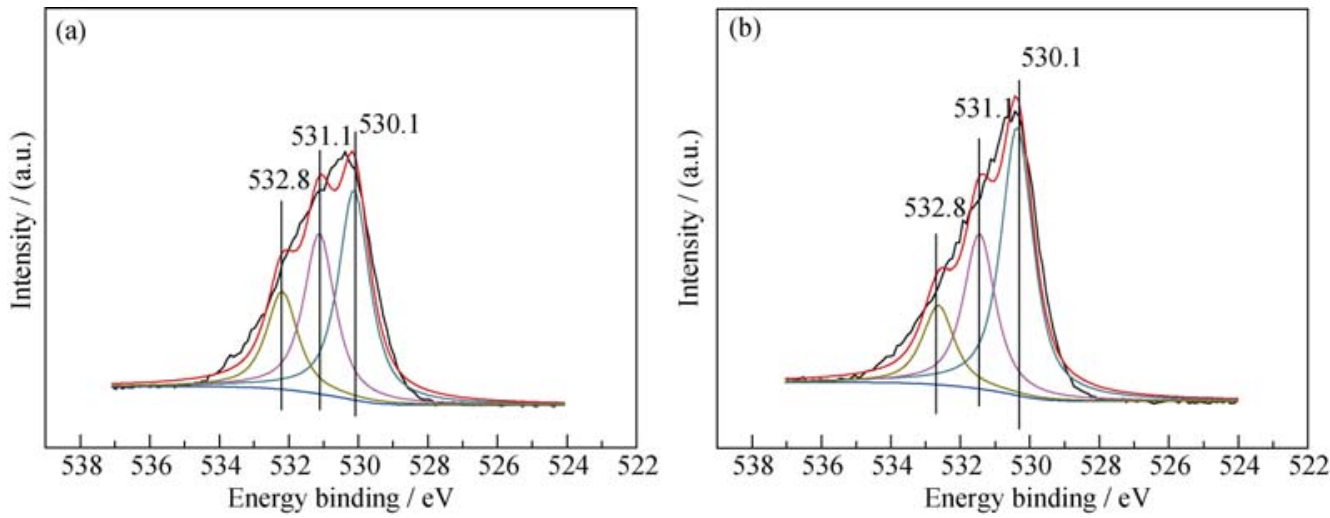

图 $4 \mathrm{Cr}-100$ 的 NO 消除性能测试前后 O1s 的 XPS 图谱

Fig. 4 XPS spectra of $\mathrm{O} 1 \mathrm{~s}$ for fresh and $\mathrm{Cr}-100$ after NO removal test (a) $\mathrm{Cr}-100$; (b) $\mathrm{Cr}-100 \mathrm{U}$

表 1 不同水热温度下制备的催化剂与经 NO 消除性能测试 失活后的催化剂的物理化学性质

Table 1 Properties and XPS result of fresh samples prepared at different hydrothermal temperatures and catalyst after NO removal test

\begin{tabular}{ccccc}
\hline Samples & $\mathrm{Cr}^{6+} / \mathrm{Cr}^{3+}$ & $\mathrm{Cr} / \mathrm{O}$ & $\mathrm{N} / \mathrm{Cr}$ & $d_{\text {XRD }} / \mathrm{nm}$ \\
\hline $\mathrm{Cr}-80$ & 0.37 & 0.22 & - & 26.4 \\
$\mathrm{Cr}-100$ & 0.64 & 0.32 & 0 & 20.7 \\
$\mathrm{Cr}-100 \mathrm{U}$ & 0.52 & 0.34 & 0.23 & 22.0 \\
$\mathrm{Cr}-160$ & 0.23 & 0.19 & - & 37.6 \\
\hline
\end{tabular}

\section{4 红外光谱分析}

图 5 为 $\mathrm{Cr}-100 \mathrm{U}$ 与 $\mathrm{Cr}-100$ 的红外峰图谱, 催化 剂 $\mathrm{Cr}-100 \mathrm{U}$ 在 1050 830 $\mathrm{cm}^{-1}$ 范围内的 $\mathrm{Cr}-\mathrm{O}-\mathrm{Cr}$ 的 特征峰消失, 并且在 $1384 \mathrm{~cm}^{-1}$ 出现硝酸根特征峰, 表明在 NO 消除测试过程中硝酸根在催化剂表面沉 积，进一步验证 XPS 对 $\mathrm{N}$ 元素的检测结果。硝酸根的 积累过程为 $\mathrm{NO}$ 经催化剂氧化成的 $\mathrm{NO}_{2}$, 与空气中存 在的少量水分反应生成硝酸根或亚硝酸根，硝酸根离 子在催化剂表面不断累积, 覆盖催化剂的活性位, 


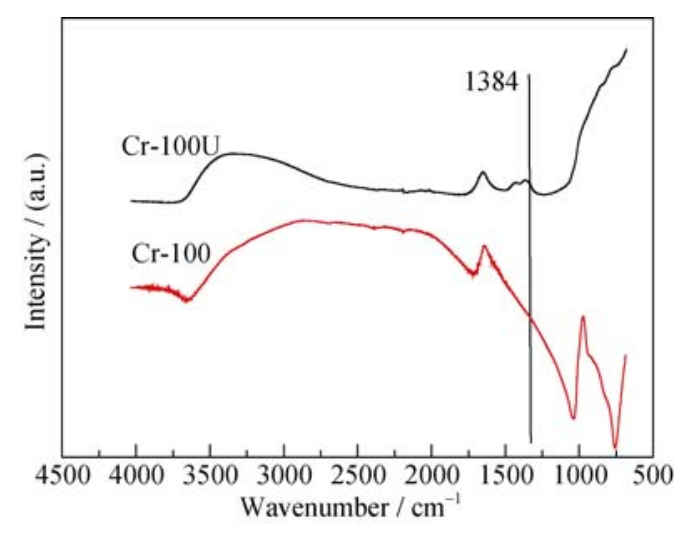

图 $5 \mathrm{Cr}-100$ 催化剂的 NO 消除性能测试前后的红外光谱

Fig. 5 FT-IR spectra of Cr-100 catalysts before and after NO removal test

导致催化剂的活性中心不断减少, $\mathrm{NO}$ 气体分子与催 化剂表面的活性中心接触困难, NO 的氧化反应便难 以进行, 导致催化剂的 NO 消除性能降低 ${ }^{[12,20]}$ 。后续 研究进一步表明不同 NO 浓度下催化剂失活快慢由 硝酸根沉积速度所决定, 较低浓度 NO 气体条件下, 其产生的硝酸根或亚硝酸根较慢, 覆盖活性位的速 度也较为缓慢, 即使 NO 消除测试进行 $24 \mathrm{~h}$ 后也没 有明显失活迹象; 处在相对较高 NO 浓度中时, 硝 酸根覆盖催化剂活性位速度远比低浓度时快, 导致 催化剂在短时间内失去催化 NO 的能力。红外表征 结果表明铬基催化剂的失活与硝酸根生成和覆盖活 性位有关。

\section{5 催化剂的活性评价}

\subsection{1 水热温度对催化剂的影响}

图 6 为不同水热温度下制备的催化剂的催化活 性曲线。由图 6(a)可以看出水热温度对催化剂活性 的影响较大, 虽然不同催化剂的初始 NO 催化氧化
性能都接近 $100 \%$, 但是随着测试时间的增加，催化 剂的活性开始下降，不同水热温度下制备的催化剂 的活性顺序为: Cr-100 $>$ Cr- $80>$ Cr-120 $>$ Cr- $140>$ Cr-160, 其中在空速 $120000 \mathrm{~mL} / \mathrm{g} \cdot \mathrm{h}$ 下, 催化剂 Cr-100 对 NO 的消除率在 $24 \mathrm{~h}$ 内始终维持在 50\%, 表现出良好的 NO 常温消除性能。图 6(b)中 Cr-100 的 $\mathrm{NO}_{2}$ 产生量较高, 证实 $\mathrm{Cr}-100$ 具有优异的 $\mathrm{NO}$ 氧 化性能。 $\mathrm{NO}_{2}$ 产生量有两个阶段的差异: $\mathrm{Cr}-100$ 的 NO 消除测试初始 $8 \mathrm{~h}$, 其 NO 常温消除率为 $100 \%$, 但在起始出口处却只检测到近 $3 \times 10^{-6}$ 的 $\mathrm{NO}_{2}$, 这可 能是催化剂表面吸附了一定量 $\mathrm{NO}_{2}$ 导致 $\left(\mathrm{NO}_{2}\right.$ 比 $\mathrm{NO}$ 更容易被催化剂所吸附)。随着 $\mathrm{NO}$ 消除测试的进行, $\mathrm{NO}_{2}$ 在催化剂表面的吸附饱和, 催化剂催化氧化成 $\mathrm{NO}_{2}$ 并不断脱附, 其浓度也随之升高, 出口处也检 测到越来越高的 $\mathrm{NO}_{2}$; $\mathrm{NO}$ 常温消除测试 $8 \mathrm{~h}$ 后, 伴 随着 Cr-100 催化剂活性的降低, $\mathrm{NO}$ 被氧化成 $\mathrm{NO}_{2}$ 的数量相应减少, 因此 $\mathrm{NO}_{2}$ 浓度开始降低。

\subsection{2 不同 NO 初始浓度对催化剂性能的影响}

除了制备条件水热温度对催化剂活性产生影响, $\mathrm{NO}$ 初始浓度也是影响催化剂活性的重要因素。图 7 为 $\mathrm{NO}$ 初始浓度分别为 $2 \times 10^{-6} 、 5 \times 10^{-6} 、 10 \times 10^{-6}$ 时, $\mathrm{Cr}-100$ 的 NO 消除性能曲线图。在 NO 浓度 $2 \times 10^{-6}$ 条件下, Cr-100 催化剂测试 $24 \mathrm{~h}$ 后仍有近 $100 \%$ 的 $\mathrm{NO}$ 消除能力; 当 NO 浓度升至 $5 \times 10^{-6}$ 时, 催化剂 $100 \%$ 消除 $\mathrm{NO}$ 的时间迅速降至 $8 \mathrm{~h}$; 当 $\mathrm{NO}$ 浓度继续 增至 $10 \times 10^{-6}$ 时, NO 气体 $100 \%$ 消除率维持只有近 $3 \mathrm{~h}$, 测试 $20 \mathrm{~h}$ 后 NO 消除率只有 $20 \%$ 。显然越高 NO 初始浓度对催化剂制约作用越显著, 这是由于高浓 度下硝酸根在催化剂表面积累速度更快, 进而影响 催化性能。
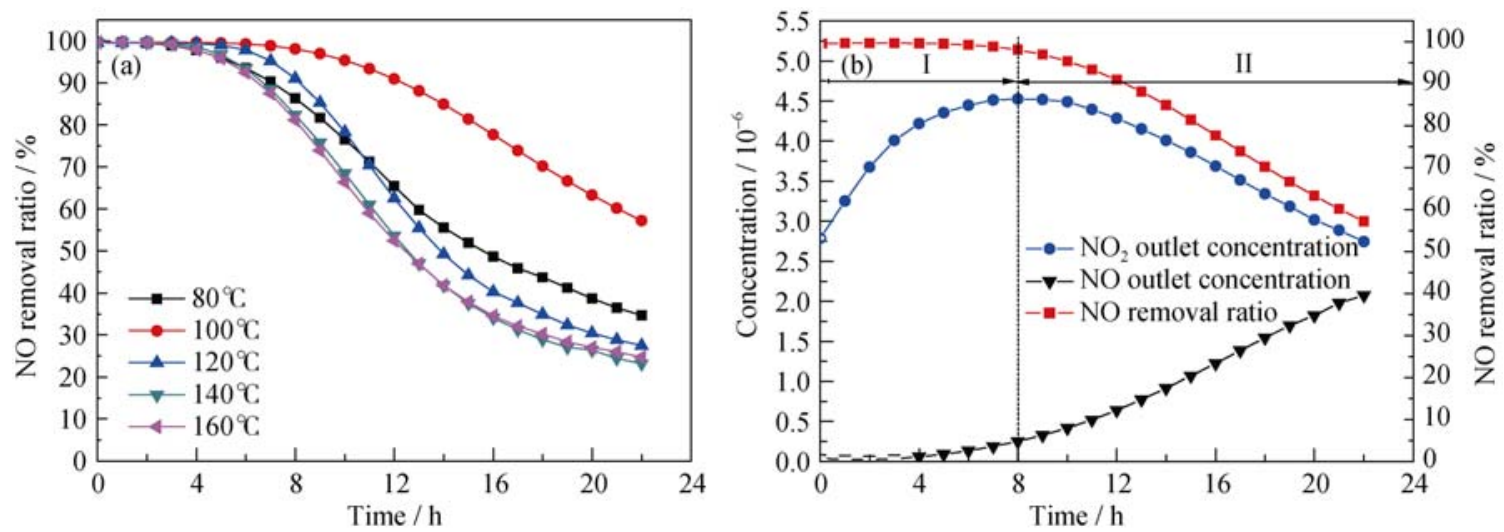

图 6 (a)不同水热温度下制备催化剂的 $\mathrm{NO}$ 消除率和(b) $\mathrm{Cr}-100$ 催化 $\mathrm{NO}$ 生成 $\mathrm{NO}_{2}$ 的量

Fig. 6 (a) The stability tests of NO oxidation at ambient temperature over chromic catalysts and (b) $\mathrm{NO}_{2}$ concentration catalyzed by Cr-100 catalyst

(Reaction conditions: mass of catalyst $=0.25 \mathrm{~g}$, total flow rate $=500 \mathrm{~mL} / \mathrm{min}, \mathrm{GHSV}=120000 \mathrm{~mL} /(\mathrm{g} \cdot \mathrm{h}), \mathrm{NO}$ concentration $=5 \times 10^{-6}$, temperature $=30^{\circ} \mathrm{C}$ ) 


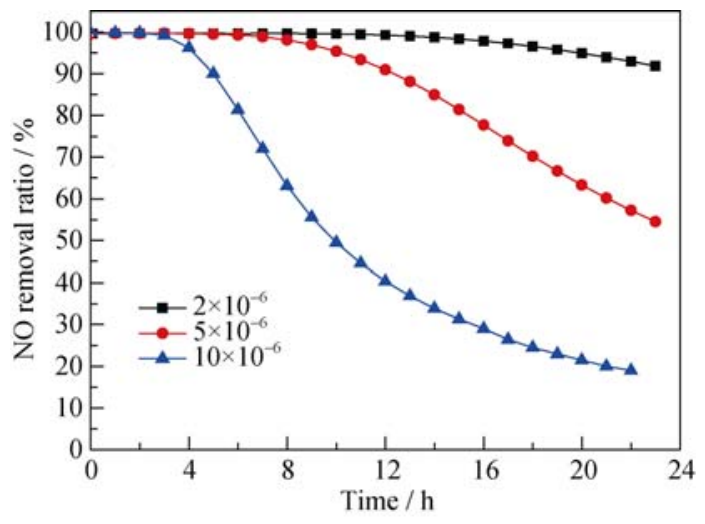

图 7 不同 NO 初始浓度对 Cr-100 催化剂的活性影响

Fig. 7 Effect of different initial NO concentrations on NO removal over the $\mathrm{Cr}-100$ catalyst

(Reaction conditions: mass of $\mathrm{Cr}-100$ catalyst $=0.25 \mathrm{~g}$, total flow rate $=$ $500 \mathrm{~mL} / \mathrm{min}, \mathrm{GHSV}=120000 \mathrm{~mL} /(\mathrm{g} \cdot \mathrm{h}), \mathrm{NO}$ concentration $=2 \times 10^{-6}$, $5 \times 10^{-6}, 10 \times 10^{-6}$, temperature $=30^{\circ} \mathrm{C}$ )

\subsubsection{Cr-100 催化剂的寿命评价}

如图 8 为 $\mathrm{Cr}-100$ 的 $\mathrm{NO}$ 常温消除寿命测试曲线, 在 $\mathrm{NO}$ 浓度为 $1 \times 10^{-6}$ 条件下, 其 $\mathrm{NO}$ 消除率维持 $90 \%$ 以上的时间近 $5 \mathrm{~d}$, 可见在较低的 NO 浓度下表 现出优异的使用寿命。但是 $5 \mathrm{~d}$ 之后催化剂失活速 度开始加快, 使用 $10 \mathrm{~d}$ 之后催化剂的 $\mathrm{NO}$ 消除率下 降到 $50 \%$ 。当混合气体中 $\mathrm{NO} / \mathrm{NO}_{2}<1, \mathrm{NO}_{x}$ 气体能够 被碱液完全吸收, 这意味着在催化剂活性降到 $50 \%$ 之前, 结合碱液的利用能够实现 $\mathrm{NO}$ 气体的常温完 全消除。

\section{3 结论}

采用水热法制备铬基催化剂, $100^{\circ} \mathrm{C}$ 水热反应 $20 \mathrm{~h}$ 条件下制备的催化剂具有最佳的 NO 催化氧化

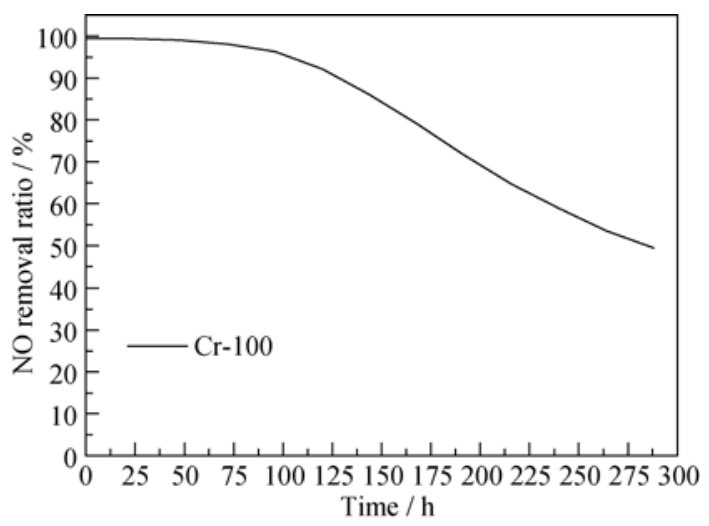

图 8 Cr-100 催化剂的 NO 常温消除寿命测试

Fig. 8 The stability tests of NO removal over Cr-100 catalyst (Reaction conditions: mass of $\mathrm{Cr}-100$ catalyst $=0.5 \mathrm{~g}$, total flow rate: $=$ $500 \mathrm{~mL} / \mathrm{min}, \mathrm{GHSV}=60000 \mathrm{~mL} /(\mathrm{g} \cdot \mathrm{h}), \mathrm{NO}$ concentration $=1 \times 10^{-6}$, temperature $=30^{\circ} \mathrm{C}$ )
性能, $60000 \mathrm{~mL} /(\mathrm{g} \cdot \mathrm{h})$ 下保持 $90 \%$ 的 NO 常温消除性 能近 $120 \mathrm{~h}$ 。催化剂表面的 $\mathrm{Cr}^{6+}$ 数量是影响催化剂 NO催化活性的重要因素, XPS 结果和活性测试结果 表明铬基催化剂对 $\mathrm{NO}$ 催化氧化性能随着其表面的 $\mathrm{Cr}^{6+} / \mathrm{Cr}^{3+}$ 增大而增强，而催化剂失活后表面 $\mathrm{Cr}^{6+} / \mathrm{Cr}^{3+}$ 出现下降。催化剂的失活主要是由于硝酸 根在催化剂表面累积, 导致催化剂活性中心被覆 盖。低 NO 浓度有利于延长催化剂的使用寿命，可 以减缓硝酸根在催化剂表面的累积速度。

\section{参考文献:}

[1] WANG H K, CHEN C H, HUANG C, et al. On-road vehicle emission inventory and its uncertainty analysis for Shanghai, China. Science of The Total Environment, 2008, 398(1): 60-67.

[2] KAMPA M, CASTANAS E. Human health effects of air pollution. Environmental Pollution, 2008, 151(2): 362-367.

[3] LIU Z M, ZHANG S X, LI J H, et al. Promoting effect of $\mathrm{MoO}_{3}$ on the $\mathrm{NO}_{x}$ reduction by $\mathrm{NH}_{3}$ over $\mathrm{CeO}_{2} / \mathrm{TiO}_{2}$ catalyst studied with in situ DRIFTS. Applied Catalysis B: Environmental, 2014, 144: $90-95$.

[4] WESTERDAHL D, WANG X, PAN X C, et al. Characterization of on-road vehicle emission factors and microenvironmental air quality in Beijing, China. Atmospheric Environment, 2009, 43(3): 697-705.

[5] CHEN Y, ZHOU X X, WANG J, et al. Synthesis of mesoporous composite oxides for the catalysis of $\mathrm{NH}_{3}$-SCR. Journal of Inorganic Materials, 2015, 30(11): 1155-1160.

[6] QIU L, MENG J J, PANG D D, et al. Reaction and characterization of $\mathrm{Co}$ and $\mathrm{Ce}$ doped $\mathrm{Mn} / \mathrm{TiO}_{2}$ catalysts for low-temperature SCR of NO with $\mathrm{NH}_{3}$.Catalysis Letters, 2015, 145(7): 1500-1509.

[7] SCHILL L, PUTLURU S S R, FEHRMANN R, et al. Lowtemperature $\mathrm{NH}_{3}-\mathrm{SCR}$ of $\mathrm{NO}$ on mesoporous $\mathrm{Mn}_{0 .}{ }_{6} \mathrm{Fe}_{0.4} / \mathrm{TiO}_{2}$ prepared by a hydrothermal method. Catalysis Letters, 2014, 144(3): 395-402.

[8] SCHILL L, PUTLURU S S R, JENSEN A D, et al. $\mathrm{MnFe} / \mathrm{Al}_{2} \mathrm{O}_{3}$ catalyst synthesized by deposition precipitation for low-temperature selective catalytic reduction of $\mathrm{NO}$ with $\mathrm{NH}_{3}$. Catalysis Letters, 2015, 145(9): 1724-1732.

[9] SOUSA J P, PEREIRA M F, FIGUEIREDO J L. NO oxidation over nitrogen doped carbon xerogels. Applied Catalysis B: Environmental, 2012, 125: 398-408.

[10] WANG MX, HUANG Z H, SHEN K, et al. Catalytically oxidation of $\mathrm{NO}$ into $\mathrm{NO}_{2}$ at room temperature by graphitized porous nanofibers. Catalysis Today, 2013, 201: 109-114.

[11] SHU Z, HUANG W M, HUA Z, et al. Template-free synthesis of mesoporous $\mathrm{X}-\mathrm{Mn}(\mathrm{X}=\mathrm{Co}, \mathrm{Ni}, \mathrm{Zn})$ bimetal oxides and catalytic application in the room temperature removal of low-concentration NO. Journal of Materials Chemistry A, 2013, 1(35): 10218-10227. 
[12] SHU Z, CHEN Y, HUANG W M, et al. Room-temperature catalytic removal of low-concentration NO over mesoporous Fe-Mn binary oxide synthesized using a template-free approach. Applied Catalysis B: Environmental, 2013, 140: 42-50.

[13] WANG J, ZHU J Z, ZHOU X X, et al. Nanoflower-like weak crystallization manganese oxide for efficient removal of low-concentration NO at room temperature. Journal of Material Chemistry A, 2015, 3(14): 7631-7638.

[14] LIU S, ZHANG M T, HUANG Y Q, et al. A novel chromic oxide catalyst for $\mathrm{NO}$ oxidation at ambient temperature. RSC Advances, 2014, 4(55): 29180-29186.

[15] HOANG D L, DITTMAR A, SCHNEIDER M, et al. Evidence of lanthanum-chromium mixed oxides formed in $\mathrm{CrO}_{x} / \mathrm{La}_{2} \mathrm{O}_{3}$ model catalysts. Thermochim Acta, 2003, 400(1): 153-163.

[16] WANG F G, ZHAO K F, ZHANG H J, et al. Low temperature CO catalytic oxidation over supported $\mathrm{Pd}-\mathrm{Cu}$ catalysts calcined at dif- ferent temperatures. Chemical Engineering Journal. 2014, 242: $10-18$.

[17] FIOL N, ESCUDERO C, VILLAESCUSA I. Chromium sorption and $\mathrm{Cr}(\mathrm{VI})$ reduction to $\mathrm{Cr}(\mathrm{III})$ by grape stalks and yohimbe bark. Bioresource Technology, 2008, 99(11): 5030-5036.

[18] YANG P, XUE X M, MENG Z H, et al. Enhanced catalytic activity and stability of Ce doping on Cr supported HZSM-5 catalysts for deep oxidation of chlorinated volatile organic compounds. Chemical Engineering Journal, 2013, 234: 203-210.

[19] LIU Z M, HAO J M, FU L X, et al. Study of $\mathrm{Ag} / \mathrm{La}_{0.6} \mathrm{Ce}_{0.4} \mathrm{CoO}_{3}$ catalysts for direct decomposition and reduction of nitrogen oxides with propene in the presence of oxygen.Applied Catalysis B: Environmental, 2003, 44(4): 355-370.

[20] LI K, TANG X L, YI H H, et al. Low-temperature catalytic oxidation of NO over Mn-Co-Ce- $\mathrm{O}_{x}$ catalyst. Chemical Engineering Journal, 2012, 192: 99-104. 